\title{
Recursion Relations for Integral Coefficients
}

\section{David A. Kosower*广}

Institut de Physique Théorique, CEA, CNRS, Université Paris-Saclay, F-91191 Gif-sur-Yvette cedex, France

E-mail: David.Kosowerdipht.fr

\section{Nicola Adriano Lo Presti}

Institute for Particle Physics Phenomenology, Durham University, Durham DH1 3LE, UK

E-mail: nicola.a.1o-prestiedurham.ac.uk

We present a recursive approach to the calculation of integral coefficients within the unitarity method for one-loop amplitudes.

13th International Symposium on Radiative Corrections

24-29 September, 2017

St. Gilden, Austria

\footnotetext{
* Speaker.

${ }^{\dagger}$ A footnote may follow.
} 


\section{Introduction}

Unitarity-based methods have brought forth a generation of numerical libraries for computing one-loop amplitudes in Yang-Mills theories [四]. These libraries are used as standard tools in computing next-to-leading order (NLO) corrections for Standard-Model processes for the LHC. There is still room for improvements in the technology used in these libraries. One line of improvement is the subject of this talk.

Such improvements to the computation of one-loop amplitudes will have an impact beyond NLO calculations. Next-to-next-to-leading order (NNLO) calculations also require the computation of one-loop amplitudes. Indeed, they require the computation of such amplitudes with one additional real emission compared to the base leading-order process, and integration over the real phase space of that emission. Accordingly, efficiency and stability improvements are important in NNLO calculations as well. The improvements we discuss should generalize to higher-loop amplitudes as well, although the generalizations may not be straightforward.

The 'gold standard' of amplitude calculations is sometimes taken to be analytic expressions, for both the integrals and their coefficients. This indeed may give the most efficient and numerically stable expressions for low-point amplitudes, but that is not the case at higher multiplicity. An analysis of the Berends-Giele recursion relations (on which we will base our developments) explains why: producing an analytic expression for a tree amplitude will (for a general helicity) yield an expression whose computational complexity grows exponentially with the number of external legs. In contrast, the complexity of a suitable numerical evaluation will grow only polynomially. This advantage arises from the fact that common sub-expressions are implicitly evaluated only once in careful numerical form.

\section{Loop Amplitudes}

We can write loop amplitudes, at one loop or beyond, in the following form,

$$
\mathrm{Ampl}=\sum_{j \in \text { Basis }} c_{j}\left(\varepsilon,\left\{\lambda_{i}, \tilde{\lambda}_{i}\right\}\right) \operatorname{Int}_{j}\left(\left\{s_{i_{1} i_{2}}\right\}\right),
$$

as a sum over a basis of integrals, each a function of the external invariants $s_{i_{1} i_{2}}$, with coefficients which are rational functions of the external spinors $\lambda_{i}$ and $\tilde{\lambda}_{i}$ in addition to the dimensional regulator $\varepsilon$. (The amplitude is computed in $D=4-2 \varepsilon$, and for Yang-Mills theories, will have a double pole in $\varepsilon$ for each order in perturbation theory.)

At one loop, the set of basis or master integrals is the well-known set of bubbles, triangles, and boxes (and tadpoles when internal particles with masses are included). At higher loops, the set of basis integrals may be determined using integration-by-parts equations [0]. These allow us to determine all algebraic identities between different integrals that may appear in the amplitude. At one loop, one can trade the dependence on $\varepsilon$ for coefficients which are $\varepsilon$-free, but where the sum in eq. (D.

\section{Coefficients}

The coefficients of the basis integrals can be computed using generalized unitarity. At one loop, once we have switched to the form of eq. ([R. $($ d) where the coefficients are free of $\varepsilon$, we can do 
this in $D=4$; computing the rational pieces requires $D=4-2 \varepsilon$. At higher loops, only the leading parts of coefficients can be computed in $D=4$.

For integrals with leading residues, the coefficients can be computed by cutting all propagators. This reduces the loop amplitude to a product of tree amplitudes. The coefficient is then given by an appropriate integral of this product of trees.

More precisely, we may view the loop amplitude as a sum over Feynman diagrams. Each loop integral that appears in the sum is taken over real momenta. If we complexify these integrals, they are multifold contours integrals, with a contour taken along the product of real slices for the different components of the loop momentum or momenta. Replacing — not deforming — this contour by one encircling the global poles of the integrand corresponds to cutting all propagators (and possibly performing additional integrals over residual degrees of freedom). These global poles are the common singularities of all cut propagators, and the contours that encircle them are multidimensional tori. In general, they are located at complex values of the loop momentum or momenta.

The formula for the coefficients has the following abstract form,

$$
c_{j}=\sum_{\text {contours } \Gamma} a_{\Gamma} \oint_{\Gamma} d \omega \prod_{d} A_{d}^{\text {tree }},
$$

where the sum is over different contours surrounding the various global poles [B]]. The weights $a_{\Gamma}$ are determined by the requirement that total derivatives give no contribution to the coefficients $c_{j}$. This requirement would be automatic for the original, real-slice, contour in the amplitude. It is nontrivial for the complex contours encircling the global poles. If we perform the integral over the degrees of freedom that enforce the cuts, we are left with contour integrals over remaining degrees of freedom,

$$
c_{j}=\sum_{\Gamma} a_{\Gamma} \oint_{\Gamma} \prod_{i} d z_{i} \operatorname{Jacobian}^{-1} \prod_{d} A_{d}^{\mathrm{tree}}\left(z_{i}\right),
$$

including the Jacobian from the cut-enforcing integrations.

\section{Laurent Expansions}

In one complex dimension, contour integration corresponds to performing a Laurent expansion, and taking the coefficient of the simple pole,

$$
\cdots+\frac{c_{2}}{z^{2}}+\frac{c_{1}}{z}+c_{0}+c_{-1} z+\cdots
$$

The analogous statement holds in higher complex dimensions, so long as we consider integrands which factorize,

$$
\frac{1}{\left(z_{1}-z_{1}^{0}\right)\left(z_{2}-z_{2}^{0}\right) \cdots}
$$

which is indeed the class of integrands to which we will restrict attention.

If we examine the simplest case, where all factors have only simple poles, we can define a Laurent expansion operator,

$$
\text { Laurent } F=\sum_{j=-1}^{\infty} F^{[-j]} z^{j}
$$


with obvious multivariate generalization,

$$
\text { Laurent } F=\sum_{j_{i}=-1}^{\infty} F^{[-\vec{\jmath}]} \prod_{i} z_{i}^{j_{i}}
$$

We can use it to peer into expansions in order to extract the global residue of a function,

$$
\begin{aligned}
& \underset{\{z\}=\left\{z^{0}\right\}}{\text { Global Residue }} \prod_{d} F_{d}(z)=\text { Laurent }\left.\prod_{d} F_{d}(z)\right|_{z^{-1}}=\prod_{d} \text { Laurent }\left.F_{d}(z)\right|_{z^{-1}}= \\
& \quad \sum_{d} F_{d}^{[1]} \prod_{r \neq d} F_{d}^{[0]}+\sum_{d_{1}, d_{2}, d_{3}} F_{d_{1}}^{[1]} F_{d_{2}}^{[1]} F_{d_{3}}^{[-1]} \prod_{r \neq d_{i}} F_{d}^{[0]}+\sum_{d_{i}} F_{d_{1}}^{[1]} F_{d_{2}}^{[1]} F_{d_{3}}^{[1]} F_{d_{4}}^{[-1]} F_{d_{5}}^{[-1]} \prod_{r \neq d_{i}} F_{d}^{[0]} \\
& \quad+\sum_{d_{i}} F_{d_{1}}^{[1]} F_{d_{2}}^{[1]} F_{d_{3}}^{[1]} F_{d_{4}}^{[-2]} \prod_{r \neq d_{i}} F_{d}^{[0]}+\cdots
\end{aligned}
$$

This expansion tells us how to calculate the residue of a product of functions, if we know the Laurent expansion of the functions individually. For example, it tells us how to calculate the residue of a product of tree amplitudes if we already know the Laurent expansion of each tree amplitude.

\section{Laurent Expansion of Amplitudes}

In order to calculate the Laurent expansion of a tree amplitude, we could imagine calculating the amplitude analytically, changing variables to the desired ones, and then performing the expansion. This would be suitable for a small number of external legs, but quickly becomes unwieldy beyond that. It also fails to be amenable to direct numerical calculation, and fails to combine common subexpressions. It would thus fail the goal of maximizing efficiency.

Instead, we seek a recursive approach. Given a recursive approach to computing the amplitudes themselves, such as the Berends-Giele recursion [四],

$$
A_{n}=f\left(A_{i}, A_{j}, \ldots\right) \quad i, j, \ldots \leq n,
$$

we seek an approach that computes the Laurent expansion of amplitudes in terms of the Laurent expansion of amplitudes with fewer external legs,

$$
\text { Laurent } A_{n}=f\left(\text { Laurent } A_{i}, \text { Laurent } A_{j}, \ldots\right) \quad i, j, \ldots \leq n .
$$

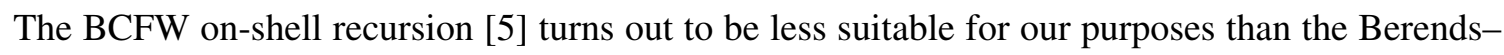
Giele one, so we will use the latter. This recursion is for the off-shell current $J_{\mu}$; we will use a form for the amputated current, without the propagator for the off-shell leg,

$$
\begin{aligned}
& J^{\mu}(1, \ldots, n)= \\
& \quad-\sum_{j=1}^{n-1} V_{3}^{\mu v^{\prime} \rho^{\prime}} \frac{d_{v^{\prime} v}}{K_{1, j}^{2}} \frac{d_{\rho^{\prime} \rho}}{K_{j+1, n}^{2}} J^{v}(1, \ldots, j) J^{\rho}(j+1, \ldots, n) \\
& -\sum_{j_{1}=1}^{n-2} \sum_{j_{2}=j_{1}+1}^{n-1} V_{4}^{\mu v^{\prime} \tau^{\prime} \rho^{\prime}} \frac{d_{v^{\prime} v}}{K_{1, j_{1}}^{2}} \frac{d_{\tau^{\prime} \tau}}{K_{j_{1}+1, j_{2}}^{2}} \frac{d_{\rho^{\prime} \rho}}{K_{j_{2}+1, n}^{2}} \\
& \quad \times J^{v}\left(1, \ldots, j_{1}\right) J^{\tau}\left(j_{1}+1, \ldots, j_{2}\right) J^{\rho}\left(j_{2}+1, \ldots, n\right)
\end{aligned}
$$


In this expressions, $d_{\mu v}$ denotes the gluon projector, $V_{3,4}$ the gluon three- and four-point vertices, while the prime on the sum indicates that vanishing denominators are omitted in the end terms, and

$$
K_{i, j}=k_{i}+\cdots k_{j}
$$

We can interchange Laurent expansion with recursion, which gives us the schematic form,

$$
\begin{aligned}
& \text { Laurent } J^{\mu}(1, \ldots, n)= \\
& -\sum_{j=1}^{n-1} \text { Laurent } V_{3}^{\mu v^{\prime} \rho^{\prime}} \text { Laurent } \frac{d_{v^{\prime} v}}{K_{1, j}^{2}} \text { Laurent } \frac{d_{\rho^{\prime} \rho}}{K_{j+1, n}^{2}} \\
& \times \text { Laurent } J^{v}(1, \ldots, j) \text { Laurent } J^{\rho}(j+1, \ldots, n) \\
& \text { - 4-pt terms. }
\end{aligned}
$$

This would ultimately lead to a system of recursion relations for the Laurent expansion coefficients $J^{[r] \mu}$,

$$
\begin{aligned}
& J^{[r] \mu}(1, \ldots, n)= \\
& \quad-\sum_{j=1}^{n-1} \sum_{r_{i}=r_{\min }(r)<0}^{1} \delta_{r_{1}+r_{2}+r_{3}+r_{4}+r_{5}} V_{3}^{\left[r+r_{1}\right] \mu v^{\prime} \rho^{\prime}}\left(\frac{d_{v^{\prime} v}}{K_{1, j}^{2}}\right)^{\left[r_{2}\right]}\left(\frac{d_{\rho^{\prime} \rho}}{K_{j+1, n}^{2}}\right)^{\left[r_{3}\right]} \\
& \quad \times J^{\left[r_{4}\right] v}(1, \ldots, j) J^{\left[r_{5}\right] \rho}(j+1, \ldots, n) \\
& \quad \text { - 4-pt terms. }
\end{aligned}
$$

\section{Example: One-Loop Triangle}

For the triangle coefficient, the maximal cut arises from performing a contour integral that cuts the three propagators, along with an additional integral over the remaining degree of freedom around the residue at infinity,

$$
\text { coeff }=\frac{1}{2 \pi i} \oint \frac{d t}{t} A_{1}(t) A_{2}(t) A_{3}(t) .
$$

The cut triangle is shown in fig. $\mathbf{m}$. We can also write this coefficient in Forde's form [圆], as

$$
-\left.\operatorname{Inf}_{t}\left(A_{1}(t) A_{2}(t) A_{3}(t)\right)\right|_{t=0}
$$

where $\operatorname{Inf}_{t}$ denotes the expansion as $t \rightarrow \infty$.

With $K_{1,2,3}$ the external momenta of the triangle as shown in fig. $\mathbb{l}$, we can define the usual mutually-projected momenta,

$$
\begin{aligned}
& K_{1}^{b, \mu}=K_{1}^{\mu}-\frac{K_{1}^{2}}{2 K_{1} \cdot K_{2}^{b}} K_{2}^{b, \mu} \\
& K_{2}^{b, \mu}=K_{2}^{\mu}-\frac{K_{2}^{2}}{2 K_{2} \cdot K_{1}^{b}} K_{1}^{b, \mu}
\end{aligned}
$$

We can then use these projected momenta to parametrize the cut loop spinors,

$$
\begin{aligned}
& \left|\ell_{i}\right\rangle=t\left|K_{2}^{b}\right\rangle+a_{i 1}\left|K_{1}^{b}\right\rangle \\
& \left.\left.\left.\mid \ell_{i}\right]=\mid K_{2}^{b}\right]+\frac{a_{i 2}}{t} \mid K_{1}^{b}\right]
\end{aligned}
$$




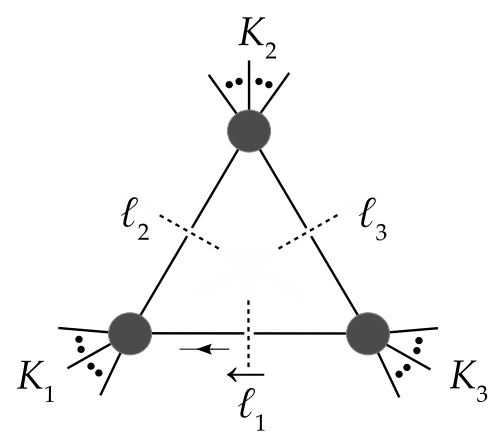

Figure 1: The triple-cut one-loop triangle integral.

At large $t$, amplitudes grow at most with $t$,

$$
A=A^{[1]} t+A^{[0]}+A^{[-1]} t^{-1}+A^{[-2]} t^{-2}+\cdots
$$

(For the $(+,+)$ helicity configuration, $A \sim t^{-3}$.) We can write down the global residue at $t=\infty$ in terms of the coefficients of different orders of the amplitudes at the three corners of the cut triangle,

$$
\begin{aligned}
- & {\left[A_{1}^{[1]} A_{2}^{[1]} A_{3}^{[-2]}+A_{1}^{[-2]} A_{2}^{[1]} A_{3}^{[1]}+A_{1}^{[1]} A_{2}^{[-2]} A_{3}^{[1]}\right.} \\
& +A_{1}^{[1]} A_{2}^{[0]} A_{3}^{[-1]}+A_{1}^{[-1]} A_{2}^{[1]} A_{3}^{[0]}+A_{1}^{[0]} A_{2}^{[-1]} A_{3}^{[1]} \\
& +A_{1}^{[0]} A_{2}^{[1]} A_{3}^{[-1]}+A_{1}^{[-1]} A_{2}^{[0]} A_{3}^{[1]}+A_{1}^{[1]} A_{2}^{[-1]} A_{3}^{[0]} \\
& \left.+A_{1}^{[0]} A_{2}^{[0]} A_{3}^{[0]}\right] .
\end{aligned}
$$

We want to find a direct approach to computing the required coefficients $A_{j}^{[i]}$.

\section{Recursion}

We will set up a system of recursion relations to compute the desired coefficients. As mentioned above, the BCFW on-shell recursion relations are not well-suited for this purpose, because we are faced either with needing to compute lower-point amplitudes with many legs carrying $t$ dependence, or cumbersome adjustments to power-counting rules (if we choose the shift legs to be $\ell_{1}$ and $\ell_{2}$ ).

It is better to use the Berends-Giele recursion relations. It's convenient to rewrite them first, however, in a purely cubic form. We can do this, for example, following Duhr, Hoeche, and Maltoni [ $[\mathbf{\square}]$ or Gleisberg and Hoeche [ [8],

$$
\begin{aligned}
J^{\mu}(1, \ldots, n) & = \\
\sum_{j=1}^{n-1}\{ & -V_{3}^{\mu v^{\prime} \rho^{\prime}} \frac{d_{v^{\prime} v}\left(K_{1, j}\right)}{K_{1, j}^{2}} \frac{d_{\rho^{\prime} \rho}\left(K_{j+1, n}\right)}{K_{j+1, n}^{2}} J^{v}(1, \ldots, j) J^{\rho}(j+1, \ldots, n) \\
& -V_{\mathrm{T}}^{\mu v^{\prime} \tau^{\prime} \rho^{\prime}} \frac{d_{v^{\prime} v}\left(K_{1, j}\right)}{K_{1, j}^{2}} d_{\rho^{\prime} \tau^{\prime} \rho \tau}\left(K_{j+1, n}\right) J^{v}(1, \ldots, j) J^{\rho \tau}(j+1, \ldots, n) \\
& \left.-V_{\mathrm{T}}^{\mu v^{\prime} \rho^{\prime} \tau^{\prime}} d_{\rho^{\prime} \tau^{\prime} \rho \tau}\left(K_{1, j}\right) \frac{d_{\nu^{\prime} v}\left(K_{j+1, n}\right)}{K_{j+1, n}^{2}} J^{\rho \tau}(1, \ldots, j) J^{v}(j+1, \ldots, n)\right\},
\end{aligned}
$$


where $J^{\mu v}$ is a fictitious-tensor current, which also has a cubic recursion,

$$
J^{\mu v}(1, \ldots, n)=V_{\mathrm{T}}^{\mu v \rho^{\prime} \tau^{\prime}} \sum_{j=1}^{n-1} \frac{d_{\rho^{\prime} \rho}\left(K_{1, j}\right)}{K_{1, j}^{2}} \frac{d_{\tau^{\prime} \tau}\left(K_{j+1}\right)}{K_{j,+1}^{2}} J^{\rho}(1, \ldots, j) J^{\tau}(j+1, \ldots, n) .
$$

In these expressions, $d_{\mu v}$ again denotes the gluon projector, $d_{\mu v \rho \tau}$ the fictitious-tensor projector, $V_{3}$ the gluon three-point vertex, and $V_{T}$ the fictitious-tensor-two gluon vertex, while the prime on the sum again indicates that vanishing denominators are omitted in the end terms. A different cubic rewriting, motivated by considerations from supersymmetry, was written down recently by Mafra and Schlotterer [Q].

One can then switch to light-cone gauge, with projector,

$$
d_{\mu \nu}^{\mathrm{LC}}(K)=-g_{\mu \nu}+\frac{q^{\mu} K^{v}+K^{\mu} q^{v}}{q \cdot K}
$$

where $q$ is the light-cone vector. We can introduce the massless flattened momentum

$$
K^{\natural}=K-\frac{K^{2}}{2 q \cdot K} q
$$

and rewrite the light-cone gauge projector in terms of on-shell polarization vectors along with an additional term,

$$
d_{\mu \nu}^{\mathrm{LC}}(K)=\sum_{\sigma= \pm} \varepsilon_{\mu}^{(\sigma)}\left(K^{b}, q\right) \varepsilon_{v}^{(\sigma) *}\left(K^{b}, q\right)+\frac{K^{2} q^{\mu} q^{v}}{\left(q \cdot K^{b}\right)^{2}} .
$$

The first two terms arise from the conventional sum over \pm helicities, while the last term can be replaced by a sum over a fictitious ' 0 ' helicity.

The non-zero vertices are $V_{3}(--+), V_{3}(-++), V_{3}(0-+)$, cyclic permutations, along with $V_{\mathrm{T}}(-+[-+])$ and $V_{\mathrm{T}}(+-[-+])$. It's convenient to absorb additional factors into the $V_{3}(0-+)$ vertex, and adjust phases to make the power-counting in $t$ cleaner.

With the shorthand notation $\langle\langle 1 \cdots n\rangle\rangle \equiv\langle 12\rangle\langle 23\rangle \cdots\langle(n-1) n\rangle$, we find the simple forms,

$$
\begin{aligned}
& J^{(+)}\left(1^{+}, \ldots, n^{+}\right)=0, \\
& J^{(-)}\left(1^{+}, \ldots, n^{+}\right)=2 i \frac{q \cdot K_{1, n} K_{1, n}^{2}}{\langle q 1\rangle\langle q n\rangle\langle\langle 1 \cdots n\rangle\rangle}, \\
& J^{(+)}\left(1^{+}, \ldots, j^{-},(j+1)^{+}, \ldots, n^{+}\right)=i \frac{K_{1, n}^{2}}{2 q \cdot K_{1, n}\langle q 1\rangle\langle q n\rangle\langle\langle 1 \cdots n\rangle\rangle}, \\
& J^{(+-)}\left(1^{-}, 2^{+}, \ldots, n^{+}\right)=-i \frac{\langle q 1\rangle^{2}\langle 1 n\rangle}{2\left\langle\langle 1 \cdots n\rangle\langle q n\rangle^{2}\right.} \\
&-i \frac{\langle q 1\rangle^{3} q \cdot K_{1, n}}{2\langle\langle 1 \cdots n\rangle\rangle\langle q n\rangle} \sum_{j=1}^{n-1} \frac{\langle j j+1\rangle}{q \cdot K_{1, j}\langle q j\rangle\langle q j+1\rangle} .
\end{aligned}
$$

Take the off-shell argument to the currents to be the cut loop momentum $-\ell_{1}$, and the on-shell legs to be $\ell_{2}, 1,2, \ldots$; only the first current in each term on the right-hand side of eq. (5.3) or (5.6) will be $t$-dependent, while the second will be just the usual tree current. 
Noting that $V_{3} \sim \mathscr{O}(t)$, while $1 /\left(\ell_{2}+K_{1, j}\right)^{2} \sim \mathscr{O}\left(t^{-1}\right)$, we obtain the following tower of recursion relations,

$$
\begin{aligned}
J^{[1]} & =\sum_{j}\left(V_{3} \frac{1}{\left(\ell_{2}+K_{1 \ldots j}\right)^{2}}\right)^{[0]} J^{[1]}\left(\ell_{2}, 1, \ldots j\right) J^{\text {tree }}(j+1, \ldots, n)+\cdots \\
J^{[0]} & =\sum_{j} \sum_{r=-1}^{0}\left(V_{3} \frac{1}{\left(\ell_{2}+K_{1 \ldots j}\right)^{2}}\right)[r] J^{[-r]}\left(\ell_{2}, 1, \ldots j\right) J^{\text {tree }}(j+1, \ldots, n)+\cdots \\
J^{[-1]} & =\sum_{j} \sum_{r=-2}^{0}\left(V_{3} \frac{1}{\left(\ell_{2}+K_{1 \ldots j}\right)^{2}}\right)^{[r]} J^{[-1-r]}\left(\ell_{2}, 1, \ldots j\right) J^{\text {tree }}(j+1, \ldots, n)+\cdots
\end{aligned}
$$

We have compared three-mass triangle coefficients computed numerically with analytic computations of Bjerrum-Bohr, Dunbar, Perkins and Warrick [ए]].

The same approach can be extended in a straightforward way to box contributions to the rational term, where the expansion is a large- $\mu^{2}$ expansion for the amplitudes, with $\mu^{2}$ representing the $D$-dimensional components of the loop momentum [ए]], and to the triangle contributions, with a double expansion in $t$ and $\mu^{2}$.

\section{References}

[1] C. F. Berger, Z. Bern, L. J. Dixon, F. Febres Cordero, D. Forde, H. Ita, D. A. Kosower and D. Maître, Phys. Rev. D 78, 036003 (2008) [arXiv:0803.4180 [hep-ph]]; G. Ossola, C. G. Papadopoulos and R. Pittau, Nucl. Phys. B 763, 147 (2007) [hep-ph/0609007]; G. Ossola, C. G. Papadopoulos and R. Pittau, JHEP 0803, 042 (2008) [0711.3596 [hep-ph]]; G. Bevilacqua, M. Czakon,

C. G. Papadopoulos, R. Pittau and M. Worek, JHEP 0909, 109 (2009) [0907.4723 [hep-ph]]; P. Mastrolia, G. Ossola, T. Reiter and F. Tramontano, JHEP 1008, 080 (2010) [arXiv:1006.0710 [hep-ph]]; G. Cullen, N. Greiner, G. Heinrich, G. Luisoni, P. Mastrolia, G. Ossola, T. Reiter and F. Tramontano, Eur. Phys. J. C 72, 1889 (2012) [arXiv:1111.2034 [hep-ph]]; S. Badger,

B. Biedermann, P. Uwer and V. Yundin, Comput. Phys. Commun. 184, 1981 (2013) [arXiv:1209.0100 [hep-ph]].

[2] F. V. Tkachov, Phys. Lett. B 100, 65 (1981); K. G. Chetyrkin and F. V. Tkachov, Nucl. Phys. B 192, 159 (1981); S. Laporta, Phys. Lett. B 504, 188 (2001) [hep-ph/0102032]; S. Laporta, Int. J. Mod. Phys. A 15, 5087 (2000) [hep-ph/0102033]; R. N. Lee, JHEP 0807, 031 (2008) [0804.3008 [hep-ph]]; C. Anastasiou and A. Lazopoulos, JHEP 0407, 046 (2004) [hep-ph/0404258]; A. V. Smirnov, JHEP 0810, 107 (2008) [0807.3243 [hep-ph]]; C. Studerus, Comput. Phys. Commun. 181, 1293 (2010) [0912.2546 [physics.comp-ph]].

[3] D. A. Kosower and K. J. Larsen, Phys. Rev. D 85, 045017 (2012) [arXiv:1108.1180 [hep-th]]; H. Johansson, D. A. Kosower and K. J. Larsen, PoS LL 2012, 066 (2012) [arXiv:1212.2132 [hep-th]].

[4] F. A. Berends and W. T. Giele, Nucl. Phys. B 306, 759 (1988).

[5] R. Britto, F. Cachazo, B. Feng and E. Witten, Phys. Rev. Lett. 94, 181602 (2005) [hep-th/0501052].

[6] D. Forde, Phys. Rev. D 75, 125019 (2007) [0704.1835 [hep-ph]].

[7] C. Duhr, S. Höche and F. Maltoni, JHEP 0608, 062 (2006) [hep-ph/0607057].

[8] T. Gleisberg and S. Höche, JHEP 0812, 039 (2008) [arXiv:0808.3674 [hep-ph]].

[9] C. R. Mafra and O. Schlotterer, JHEP 1603, 097 (2016) [arXiv:1510.08846 [hep-th]]. 
[10] N. E. J. Bjerrum-Bohr, D. C. Dunbar and W. B. Perkins, JHEP 0804 (2008) 038 [arXiv:0709.2086 [hep-ph]]; D. C. Dunbar, W. B. Perkins and E. Warrick, JHEP 0906 (2009) 056 [arXiv:0903.1751 [hep-ph]].

[11] S. D. Badger, JHEP 0901, 049 (2009) [arXiv:0806.4600 [hep-ph]]. 